\title{
QUANTITATIVE STUDIES ON HUMAN URINARY METABOLITES OF TRYPTOPHAN AS AFFECTED BY ISONIAZID AND DEOXYPYRIDOXINE ${ }^{1}$
}

\author{
BY J. M. PRICE, 2 R. R. BROWN, AND FRANK C. LARSON \\ (From the Cancer Research Hospital, Medical School, University of Wisconsin, and the \\ Veterans Administration Hospital, Madison, Wisc.)
}

(Submitted for publication May 21, 1957; accepted July 25, 1957)

Peripheral neuropathy has been observed as a complication of the treatment of tuberculosis with isonicotinic acid hydrazide (isoniazid) $(1,2)$, especially when large doses have been employed (3, 4). This complication of isoniazid therapy has been largely eliminated by the simultaneous administration of pyridoxine $(3,5)$.

In pyridoxine deficiency, rats (6) and humans (7) have been found to excrete abnormally large amounts of the tryptophan metabolite xanthurenic acid. The administration of the pyridoxine antagonist, deoxypyridoxine, to humans (8) or rats (9) has been shown to produce a severe disturbance of tryptophan metabolism in these species as manifested by the excretion of large amounts of xanthurenic acid following the administration of a "loading" dose of tryptophan. These observations have prompted studies of the urinary excretion of xanthurenic acid as an index of the antipyridoxine activity of isoniazid. Studies with humans (3) and rats (10) ingesting isoniazid have revealed an increased excretion of xanthurenic acid after ingestion of tryptophan. Rosen (11) reported no increase in xanthurenic acid excretion when isoniazid was administered to pyridoxine-deficient rats, even though the rats showed other signs of pyridoxine deficiency. In the studies of Biehl and Vilter (3) the administration of large doses of isoniazid resulted in a considerable increase in urinary excretion of vitamin $B_{6}$, about a 40 per cent incidence of clinical evidence for pyridoxine deficiency, but an irregular elevation of xanthurenic acid excretion following a tryptophan load. Thus

\footnotetext{
1 Supported in part by grants from the American Cancer Society, upon recommendation of the Committee on Growth of the National Research Council; the Wisconsin Division of the American Cancer Society; the Elsa U. Pardee Foundation; and the National League Baseball Club of Milwaukee, Inc.

2 Scholar in Cancer Research of the American Cancer Society.
}

it appeared that the clinical evidence for pyridoxine deficiency in rats or humans given isoniazid was more striking than the xanthurenic acid excretion studies would suggest $(3,11)$.

The present studies were undertaken in an effort to determine whether or not the simultaneous quantitative measurement of several urinary metabolites of tryptophan would reveal additional evidence of a disturbance in the metabolism of this essential amino acid in patients ingesting isoniazid. The extensive paper chromatographic studies of Dalgliesh (12) revealed that pyridoxine-deficient rats excreted not only xanthurenic acid, but large amounts of several other intermediary metabolites of tryptophan. Methods have been developed for the quantitative determination of several of these intermediary metabolites of tryptophan in urine (13-15). The application of these methods in quantitative studies of tryptophan metabolism in normal human subjects has been reported $(14,16)$.

The present detailed studies on tryptophan metabolism were made repeatedly over a period of several weeks during which time the dosage of isoniazid was increased until abnormal tryptophan metabolism was observed. Once the metabolic pattern became abnormal, pyridoxine and three other members of the vitamin B-complex, each of which has been suggested to play some role in tryptophan metabolism, were administered in an attempt to determine which of these vitamins might be required to overcome the effects of isoniazid on this metabolic pathway. A preliminary report of these studies has been made (17).

\section{EXPERIMENTAL}

Analytical procedures. The methods for the determinations of urinary kynurenic acid, xanthurenic acid, $N$-methyl-2-pyridone-5-carboxamide (pyridone), anthranilic acid glucuronide, $N$ - $\alpha$-acetylkynurenine, kynurenine, o-aminohippuric acid, and 3-hydroxykynurenine were those previously described (13-15). Paper chromatog- 


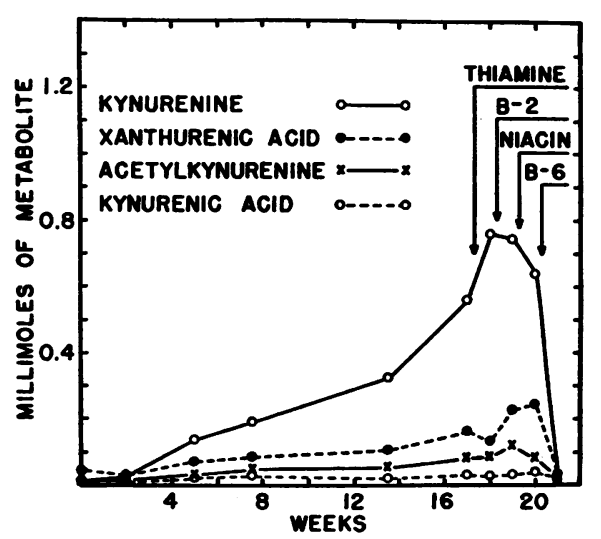

Fig. 1. Minlimoles of Metabolites Excreted in the 24-hour PERIOd Following Ingestion OF TRYPTOPHAN

Subject 1 (weight, $77 \mathrm{Kg}$.) was receiving $300 \mathrm{mg}$. of isoniazid daily at the start of the experiment. The dose was increased to $600 \mathrm{mg}$. after two weeks and was given at a level of $750 \mathrm{mg}$. daily from the start of the sixth week until the end of the experiment. B-2= riboflavin; B-6 = pyridoxine hydrochloride.

raphy $(14,18)$ was utilized to verify the quantitative determinations of aromatic amines in every urine studied.

Subjects. The studies were conducted on six male patients with tuberculosis from the Veterans Administration Hospital, but the detailed data presented were obtained from three of these subjects. Subject 2 was used for three of the studies and the others were used for one study each. Subjects 1 and 2 were Negroes, and subject 3 was white. The subjects all had pulmonary tu-

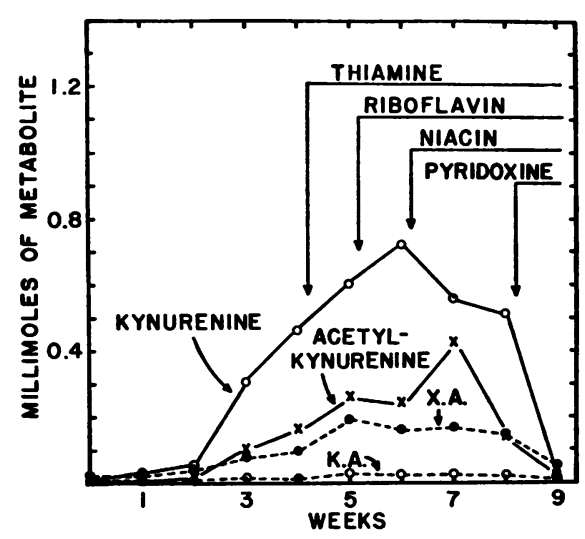

Fig. 2. Millimoles of Metabolites Excreted in the 24-hour Period Following Ingestion of Tryptophan

Subject 2 (weight, $57 \mathrm{Kg}$.) was not on therapy at the start of the experiment. Isoniazid was started at a level of $\mathbf{3 0 0} \mathrm{mg}$. daily after the initial study and increased to $450,600,750 \mathrm{mg}$. daily after the first, second, and third week, respectively, and continued throughout the study at a level of $750 \mathrm{mg}$. daily. X. A. = xanthurenic acid; K. A. = kynurenic acid.

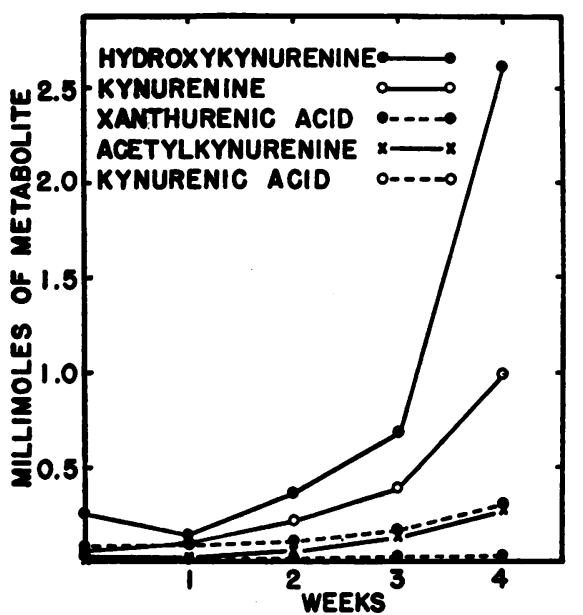

Fig. 3. Millimoles of Metabolites Excreted IN THE 24-HOUR PERIOD Following INGESTION OF TrYPTOPHAN

Subject 3 (weight, $51 \mathrm{Kg}$ ) was started on $300 \mathrm{mg}$. of isoniazid daily after the initial, zero-time study and the dose was increased to 450,600 , and $900 \mathrm{mg}$. daily after the first, second, and third week, respectively. This study was discontinued because the patient left the hospital after the fourth week. Note that the scale of this graph is different from Figures 1 and 2.

berculosis but were in a stationary phase of their disease. The subjects were ambulant and afebrile. Genitourinary tuberculosis was specificially excluded.

Each patient was thoroughly instructed concerning the proposed experiments. At weekly intervals (except

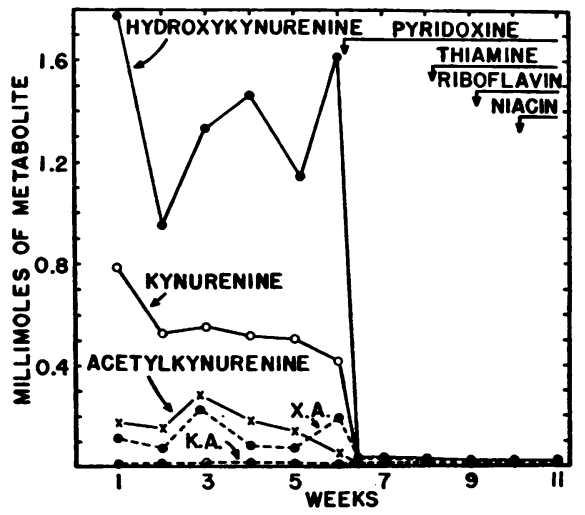

Fig. 4. Minlimoles of Metabolites Excreted in the 24-hour Period Following Ingestion of Tryptophan

Subject 2 was given $900 \mathrm{mg}$. of isoniazid daily starting at zero time, but had not received any chemotherapy for 18 weeks prior to the time this study was started. No pre-treatment study was done, but the patient had a normal pre-treatment response to the $2 \mathrm{Gm}$. of tryptophan in the other experiments in which he participated (Figures 2 and 5). X. A. =xanthurenic acid; K. A. = kynurenic acid. 


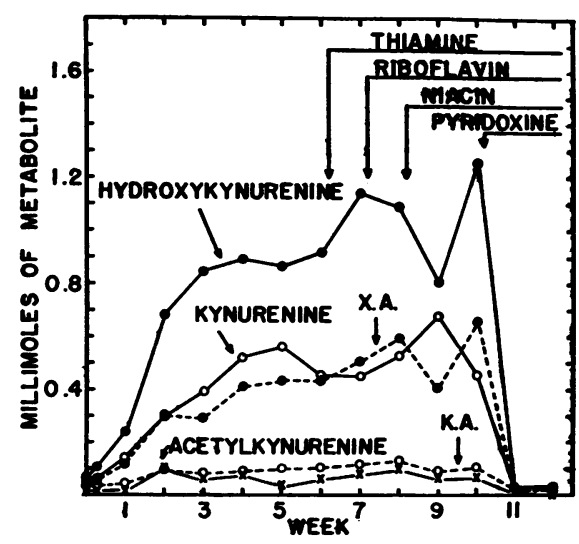

Fig. 5. Minlmoles of Metabolites Excreted in the 24-hour Period Following Ingestion of TryPtophan

Deoxypyridoxine was given to subject 2 at a level of $75,150,225,300$, and $375 \mathrm{mg}$. daily starting after 0,1 , 2,3 , and 5 weeks, respectively, and the latter dose was continued throughout the study. X. A.= xanthurenic acid; K. A. = kynurenic acid.

for Figure 1) the following procedure was carried out: 2 24-hour urine collection (in amber bottles under toluene) was started at $7 \mathrm{a.m}$. on Monday, and the subject ingested $2.0 \mathrm{Gm}$. (9.8 mM) of L-tryptophan at 7 a.m. on Tuesday. A second 24-hour urine collection was completed by 7 a.m. on Wednesday and all changes in medication were made at this time.

The tryptophan was administered as a single dose in four $0.5-\mathrm{Gm}$. gelatin capsules (Figures 1 and 2) or as four $0.5-\mathrm{Gm}$. tablets ${ }^{3}$ (Figures 3 through 5 ). The deoxypyridoxine 4 was given in gelatin capsules and the isoniazid and vitamins were standard pharmaceutical preparations. The deoxypyridoxine and isoniazid were administered in three equal doses each day and in amounts indicated in the legends for the figures. Thiamine was given at a dosage of $300 \mathrm{mg}$., and the other vitamins at levels of $75 \mathrm{mg}$., in three equal portions daily. Once a vitamin supplement was started it was continued throughout the remainder of the study. The urine samples were always analyzed the week they were collected in order to obtain the data in time so that it could be used in selecting the next dose of drug or other supplement.

\section{RESULTS}

The data obtained have been summarized in Figures 1 through 5. An abbreviated outline of the metabolic pathway from tryptophan to nicotinic acid has been presented in Figure 6. The

${ }^{3}$ The tryptophan was compressed into $0.5-\mathrm{Gm}$. tablets for these studies by Dr. Rodney P. Gwinn and his associates at Abbott Laboratories, North Chicago, Illinois.

- The deoxypyridoxine was generously supplied by Dr. Elmer Severinghaus of Hoffmann-La Roche, Inc., Nutley, New Jersey. positions of the metabolites under consideration in these studies of the pathway have all been included in the diagram. The data for pyridone, anthranilic acid, anthranilic acid glucuronide, and o-aminohippuric acid were not shown on the graphs because the values remained at normal or less than normal levels throughout the studies. The basal excretions (the 24-hour periods before the administration of tryptophan) of the metabolites represented in Figures 1 through 5 were very low compared with excretions after ingestion of the $9.8 \mathrm{mM}$ supplements of tryptophan. Therefore, the basal values were not subtracted from the data presented in the graphs. Thus the data in Figures 1 through 5 represent the millimoles of metabolites excreted in the 24-hour period following ingestion of the $9.8 \mathrm{mM}$ of tryptophan (Figure 1). Unless otherwise indicated all values below refer to the 24 hours after tryptophan.

Although subject 1 (Figure 1) was receiving $300 \mathrm{mg}$. of isoniazid daily at the start of the experiment, the urinary excretion of metabolites was normal for the first two studies. After three weeks

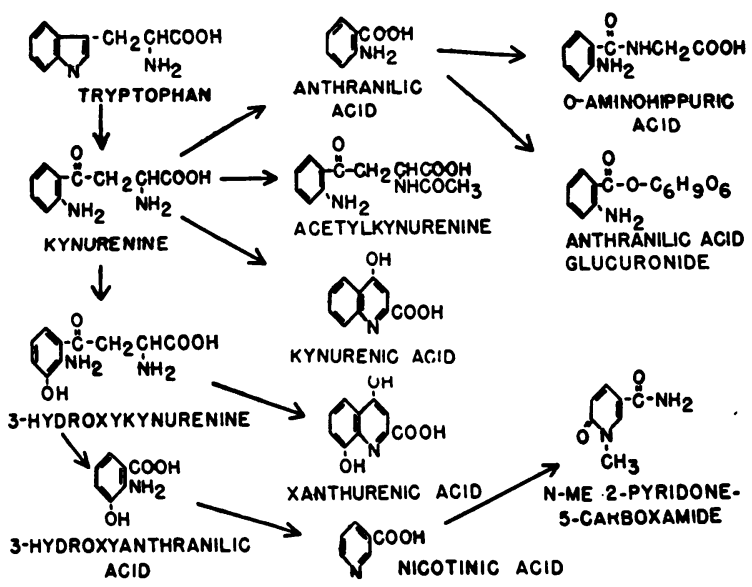

Fig. 6. An Abrreviated Diagram of the Metabolic Pathway From Tryptophan to Nicotinic Acid and $N$-methyl-2-Pyridone-5-CarboXakide, the ChIEF URInary Niacin Metabolite in Man

Pyridoxine, in the form of pyridoxal phosphate, has been reported to function in the conversion of : 1) $\mathbf{k y}$ nurenine to anthranilic acid $(36)$; 2) 3-hydroxykynurenine to 3-hydroxyanthranilic acid $(37)$; 3) kynurenine to kynurenic acid (37); and 4) 3-hydroxykynurenine to xanthurenic acid (37). Niacin in the form of triphosphopyridine nucleotide was required for the hydroxylation of kynurenine to hydroxykynurenine (18) and riboflavin also has been suggested to play a role in this reaction (24-26). 
of ingestion of $600 \mathrm{mg}$. of isoniazid daily, the excretion of kynurenine and acetylkynurenine were increased to abnormal levels after ingestion of tryptophan. After the isoniazid intake was increased to $750 \mathrm{mg}$. daily the yield of kynurenine, xanthurenic acid and acetylkynurenine rose to increasingly abnormal levels. The vitamin supplements were started after the seventeenth week and an additional vitamin was added weekly. After the pyridoxine was added to the vitamin supplement the response to the test dose of tryptophan was restored to normal. The effects of the other three vitamin supplements were of doubtful significance, though there was a slight drop in kynurenine excretion when the niacin supplement was started.

The second experiment (Figure 2) was essentially $a$ repetition of the first study and gave comparable results. Subject 2 did not receive any isoniazid when the initial study was done. This subject usually excreted more acetylkynurenine than xanthurenic acid, while the opposite was true with subject 1 . The subject was kept on supplements of thiamine, riboflavin, and nicotinamide for two weeks before the pyridoxine was started, since the results obtained with the first subject suggested that a slight decrease in kynurenine occurred after niacin was started. Again the addition of niacin slightly decreased the yield of kynurenine. The pyridoxine supplements restored the tryptophan metabolism to normal.

By the time the next study was undertaken (Figure 3) a method for the determination of 3-hydroxykynurenine was available. This subject excreted an abnormally high level of 3-hydroxykynurenine in the initial study. A daily administration of 450 to $600 \mathrm{mg}$. of isoniazid resulted in a severe disturbance of tryptophan metabolism and the last study at four weeks (900 mg. isoniazid daily) revealed a remarkable increase in the excretion of 3-hydroxykynurenine. This one metabolite accounted for over 25 per cent of the $9.8 \mathrm{mM}$ supplement of tryptophan, and the kynurenine accounted for over 10 per cent of the amino acid given. Acetylkynurenine and xanthurenic acid were excreted in approximately equal quantities, and as in all studies on isoniazid the kynurenic acid excretion was less than normal.

In the next study (Figure 4) the subject was started on $900 \mathrm{mg}$. of isoniazid daily at zero time and was maintained at that dosage for eleven weeks. Within one week, when the first study was made, the hydroxykynurenine accounted for about 17 per cent of the dose of tryptophan. After six weeks on an intake of $900 \mathrm{mg}$. of isoniazid daily the pyridoxine supplements were started, and thereafter the response to the $9.8 \mathrm{mM}$ dose of tryptophan was normal. The addition of the other three vitamins had no detectable influence on the response to tryptophan.

The effect on deoxypyridoxine on the fate of ingested tryptophan (Figure 5) was similar to that of isoniazid except that xanthurenic acid excretion was considerably greater and was almost equal to that of kynurenine. Unlike the observations during isoniazid treatment the administration of deoxypyridoxine was accompanied by abnormally high kynurenic acid excretion. Again, although the addition of thiamine, riboflavin and niacin supplements had little, if any, effect on the metabolism of the test dose of tryptophan, pyridoxine restored the urinary excretion of metabolites to normal.

Another subject was started on deoxypyridoxine (subject 3) at a level of $150 \mathrm{mg}$. daily. Within one week the response to tryptophan ingestion was similar to that in Figure 5 at the same dosage of deoxypyridoxine (2 weeks). The first supply of deoxypyridoxine was exhausted at this point and a new batch was used. The second week (end of one week on the new supply) the response to tryptophan was about half that of the previous week. Although the intake of deoxypyridoxine was gradually increased to $450 \mathrm{mg}$. daily the excretion of the metabolites of tryptophan fell to almost normal by the seventh week, indicating that the second lot of deoxypyridoxine probably contained some substance with vitamin $B_{6}$ activity."

The other studies on isoniazid which were not presented were all incomplete for some reason, usually because the subject developed some additional illness. The incomplete results were in general agreement with the data presented (Figures 1 through 4).

None of the subjects experienced any symp-

\footnotetext{
5 Hoffmann-La Roche, Inc, reported that samples of the two lots of deoxypyridoxine have now been tested microbiologically for antipyridoxine activity, and the first batch of drug had about 20 times the activity of the second.
} 
toms that could be attributed to the ingestion of either isoniazid or deoxypyridoxine.

Although, as stated previously, the basal excretions of the various metabolites (the 24-hour periods prior to tryptophan supplementation) were of insignificant quantities compared with the excretion following ingestion of tryptophan, they were altered somewhat by isoniazid or deoxypyridoxine. Thus, kynurenic acid was usually excreted at basal levels of 7 to $10 \mu \mathrm{M}$ daily and the ingestion of isoniazid or deoxypyridoxine decreased these levels to 3 to $8 \mu \mathrm{M}$ per day. Basal values for kynurenine excretion ranged from 8 to $12 \mu \mathrm{M}$ daily but invariably increased to 35 to $45 \mu \mathrm{M}$ daily at the times when the response to the tryptophan load yielded quantities of 700 to $1,000 \mu \mathrm{M}$ of this metabolite. The pre- and post-tryptophan values for urinary o-aminohippuric acid and anthranilic acid glucuronide were very similar to those observed in studies on normal human subjects (14), and the volatile amine from the acetylkynurenine fraction (14) was usually comparable to the values found on direct diazotization and coupling of this fraction, which indicated that little, if any, free anthranilic acid was excreted.

Although the pyridone excretion usually remains elevated for several days after the ingestion of tryptophan $(14,16)$ or niacin $(19)$ the amount excreted the first day provides some indication of niacin formation from tryptophan. In general, the subjects excreted less than the expected amounts of pyridone following ingestion of tryptophan. Subject 1, however, invariably excreted more than the expected amounts of pyridone following ingestion of tryptophan, so that in these studies no conclusions could be made concerning the effects of isoniazid on the conversion of tryptophan to niacin. When the nicotinamide supplements were given to these subjects the pyridone excretion increased to very high levels, which suggested that isoniazid and deoxypyridoxine had little, if any, effect on the conversion of niacin to the pyridone.

\section{DISCUSSION}

In these studies the subjects were maintained on a general hospital diet and no attempt was made to regulate the food intake. The subjects maintained their weights or gained slightly during the experiments. Previous work has indicated that the urinary excretion of these metabolites of tryptophan (except for the pyridone) was about the same on self-selected or constant dietary intake (16). The magnitude of the disturbance of tryptophan metabolism observed in the present studies makes it appear unlikely that a carefully regulated diet would have contributed much to the results. However, a larger dietary intake of pyridoxine probably would have made it necessary to administer higher doses of isoniazid and deoxypyridoxine to achieve effects similar to those observed.

All of the subjects used in these studies had essentially normal tryptophan metabolism prior to the ingestion of isoniazid or deoxypyridoxine except for subject 3 who, for unknown reasons, excreted an abnormal amount of 3-hydroxykynurenine. Abnormal tryptophan metabolism has been found in patients with tuberculosis $(20,21)$. However, Dalgliesh and Tekman (22) reported that large quantities of kynurenine and hydroxykynurenine were excreted by patients, including those with tuberculosis, only if there was an elevation of body temperature. The fact that the subjects used in the present studies did not have an elevation of the body temperature and had normal tryptophan metabolism in the absence of the drugs corroborates these findings.

Biehl and Vilter (3) reported that not all tuberculous patients receiving isoniazid therapy excreted abnormal quantities of xanthurenic acid after tryptophan loading. The present studies, however, leave little doubt that large doses of isoniazid produced a profound disturbance in the metabolism of a test dose of tryptophan. Normal subjects excreted an additional 3 to $5 \mu \mathrm{M}$ of acetylkynurenine and 15 to $30 \mu \mathrm{M}$ of kynurenine during the first 24 hours after ingestion of $9.8 \mathrm{mM}$ of L-tryptophan $(14,16)$. In subjects ingesting isoniazid, however, the yield of acetylkynurenine reached 20 to 55 times, and of kynurenine 20 to 30 times, these quantities. Xanthurenic acid excretion was not nearly as useful as the excretion of kynurenine or hydroxykynurenine in detecting inability to metabolize tryptophan in a normal manner, and kynurenic acid excretion was usually normal or less than normal in these subjects. There appear to be some clinical conditions where the urinary excretion of xanthurenic acid may be normal or less than normal after ingestion of a 
"loading dose" of tryptophan, while kynurenic acid, kynurenine, and other metabolites may be found in very abnormally high levels (23). Thus the determination of a single urinary metabolite of tryptophan cannot be relied upon as an indication of abnormal tryptophan metabolism.

Pyridoxine appeared to correct the metabolism of tryptophan without any definite evidence for the need of additional thiamine, riboflavin, or niacin. Niacin in the form of triphosphopyridinenucleotide has been shown to be required for the hydroxylation of L-kynurenine to 3-hydroxy-L-kynurenine (18). There was some evidence that niacin administration slightly decreased the excretion of kynurenine, but since hydroxykynurenine was not determined in these studies (Figures 1 and 2) it was not known whether or not these observations indicated increased hydroxylation of kynurenine. Considerable hydroxykynurenine was excreted by the subjects ingesting the largest doses of isoniazid (Figures 3 and 4 ) which would indicate that in this one reaction the isoniazid failed to reveal significant antiniacin activity.

Riboflavin supplements were administered to these subjects because it has been reported (24 27) that this vitamin may be involved in the metabolism of tryptophan. Dalgliesh (28) found that rats deficient in both pyridoxine and thiamine failed to excrete the urinary metabolites of tryptophan which were detected in pyridoxine deficiency, and suggested that thiamine may be involved in the formation of formylkynurenine from tryptophan. In the present experiments there was no demonstrable effect of riboflavin supplements on tryptophan metabolism. A slight increase in kynurenine excretion occurred after the thiamine supplements were started, but this was observed under conditions where the kynurenine excretion was increasing, probably because of the administration of the drugs.

In previous studies (16) conducted in a manner similar to those reported here, but with normal subjects on a constant dietary regimen, it was found that the increase in urinary tryptophan metabolites after supplementation with $9.8 \mathrm{mM}$ of the amino acid would account for about 2 per cent of the administered tryptophan. Slightly less than half of the tryptophan was accounted for as the pyridone metabolite of niacin. Such results might be interpreted to indicate that very little tryptophan enters the kynurenine pathway under normal conditions. In the present experiments as much as 40 per cent of the $9.8 \mathrm{mM}$ dose of tryptophan was accounted for in the urine in the form of kynurenine and its metabolites. Thus, subjects ingesting isoniazid either converted more than normal amounts of tryptophan to kynurenine and related substances, or they were unable to convert these intermediates to the usual end products at a normal rate.

The enzyme system tryptophan peroxidase, which initiates the conversion of tryptophan to kynurenine, has been shown to be adaptive (29). Administration of substances like kynurenine, histamine, phenylalanine, cortisone, and especially tryptophan itself have been found to increase the activity of tryptophan peroxidase in rat liver (29). Thus it may be possible that patients ingesting isoniazid convert more than the normal quantity of tryptophan to kynurenine. However, since there is so much evidence to indicate that isoniazid has an antagonistic effect on pyridoxine in man $(3,5)$ and the metabolites found in large quantities in the urine in the present studies were identical with those detected in the urine of pyridoxinedeficient rats (12), it appears reasonable to explain the present findings in terms of incomplete metabolism of the kynurenine produced. Dalgliesh and Tabechian (30) have recently discussed the metabolic fate of tryptophan and have suggested that considerable tryptophan may normally enter this pathway and may be converted to 3-hydroxyanthranilic acid and then to non-aromatic products. This conclusion was reached by eliminating other metabolites of kynurenine, such as kynurenic and xanthurenic acids, as likely precursors of unknown major metabolites because they appeared to be relatively inert from a metabolic standpoint, while 3-hydroxyanthranilic acid has been shown to be metabolized rapidly (31-33). Although it is now known that kynurenic acid and xanthurenic acid cannot be regarded as metabolically inert, since both have been shown to be dehydroxylated in the 4-position $(34,35)$, there is little evidence to suggest that these quinoline derivatives are excreted in quantities adequate to account for the majority of supplements of L-tryptophan. The results of the present study, therefore, cannot be regarded as in disagreement with the explanation offered by Dalgliesh and Tabechian (30) for the 
normal metabolic fate of the major part of a tryptophan supplement.

The differences in the patterns of the urimary metabolites produced by isoniazid and deoxypyridoxine were striking. Xanthurenic and kynurenic acids were both excreted in abnormally large quantities during ingestion of deoxypyridoxine, while less than normal amounts of kynurenic acid and only moderately increased quantities of xanthurenic acid were excreted under the influence of isoniazid. Since kynurenine and hydroxykynurenine were produced in large amounts in the presence of each drug it would appear that in vivo isoniazid was a better inhibitor of kynurenine transaminase than deoxypyridoxine.

\section{SUMMARY}

The influence of isoniazid and deoxypyridoxine on the urinary excretion of tryptophan metabolites by tuberculous patients was studied. The subjects all had essentially normal tryptophan metabolism prior to treatment with these drugs.

During treatment with isoniazid or deoxypyridoxine, 3-hydroxykynurenine was excreted in the urine in quantities adequate to account for from 10 to 25 per cent of a $9.8 \mathrm{mM}(2 \mathrm{Gm}$.) supplement of L-tryptophan. Kynurenine was often excreted in quantities equivalent to 5 to 10 per cent of the tryptophan administered, while $N$ - $\alpha$-acetylkymurenine and xanthurenic acid were also excreted in abnormally large amounts. The excretion of kynurenic acid was slightly less than normal during isoniazid treatment and abnormally high when deoxypyridoxine was administered. Normal or slightly less than normal amounts of o-aminohippuric acid, anthranilic acid glucuronide, anthranilic acid and $N$-methyl-2-pyridone-5-carboxamide were found in the urine after ingestion of tryptophan during treatment with either drug.

Pyridoxine administration resulted in a return to normal tryptophan metabolism even when the ingestion of isoniazid or deoxypyridoxine was continued. Supplemental thiamine, riboflavin, or niacin had little, if any, effect on the abnormal tryptophan metabolism produced by these drugs.

\section{ACKNOWLEDGMENT}

The authors are indebted to Mrs. Mary Bryan, Mrs. Marian Prahl, and Mrs. Masako Kaihara for assistance with the amalytical procedurea.

\section{REFERENCES}

1. Jones, W. A., and Jones, G. P., Peripheral neuropathy due to isoniazid: report of two cases. Lancet, 1953, 1, 1073.

2. Biehl, J. P., and Skavlem, J. H., Toxicity of isoniazid. Am. Rev. Tuberc., 1953, 68, 296.

3. Biehl, J. $P_{.2}$ and Vilter, R. W., Effect of isoniazid on vitamin $B_{6}$ metabolism; its possible significance in producing isoniazid neuritis. Proc. Soc. Exper. Biol. \& Med., 1954, 85, 389.

4. Lubing, H. N., Peripheral neuropathy in tuberculosis patients treated with isoniazid. Am. Rev. Tuberc., 1953, 68, 458.

5. Carlson, H. B., Anthony, E. M., Russell, W. F., Jr., and Middlebrook, G., Prophylaxis of isoniazid neuropathy with pyridoxine. New England J. Med, 1956, 255, 118.

6. Lepkovsky, S., Raboz, E., and Haagen-Smit, A. J., Xanthurenic acid and its role in the tryptophane metabolism of pyridoxine-deficient rats. J. Biol. Chem., 1943, 149, 195.

7. Greenberg, L. D, Bahr, D. F, McGrath, H., and Rinehart, J. F., Xanthurenic acid excretion in the human subject on a pyridoxine-deficient diet. Arch. Biochem., 1949, 21, 237.

8. Glazer, H. S., Mueller, J. F., Thompson, C., Hawkins, V. R., and Vilter, R. W., A study of urinary excretion of xanthurenic acid and other tryptophan metabolites in human beings with pyridoxine deficiency induced by desoxypyridoxine. Arch. Biochem., 1951, 33, 243.

9. Porter, C. C., Clark, I., and Silber, R. H., The effect of pyridoxine analogues on tryptophane metabolism in the rat. J. Biol. Chem, 1947, 167, 573.

10. Boone, I. U., Turney, D. F., and Langham, W. H., Isoniazid in vitamin $B_{0}$-deficient rats. J. Lab. \& Clin. Med., 1955, 46, 549.

11. Rosen, F., Effect of isonicotinic acid hydrazide on niacin and pyridoxine metabolism in rats. Proc. Soc. Exper. Biol. \& Med, 1955, 88, 243.

12. Dalgliesh, C. E., The relation between pyridoxin and tryptophan metabolism, studied in the rat. Biochem. J., 1952, 52, 3.

13. Price, J. M., The determination of $N$-mèthyl-2-pyridone-5-carboccamide in human urine. J. Bial. Chem., 1954, 211, 117.

14. Brown, R. R., and Price, J. M., Quantitative studies on metabolites of tryptophan in the urine of the dag, cat, rat, and man. J. Biol. Chem., 1956, 219, 985.

15. Brown, R. R., The isolation and determination of urinary hydroxykymurenine. J. Biol. Chem., 1957, 227, 649.

16. Price, J. M., Brown, R. R., and Ellis, M. E, Quantitative studies on the urinary excretion of tryptophan metabolites by humans ingesting a constant diet. J. Nutrition, 1956, 60, 323.

17. Price, J. M., Brown, R. R., and Larson, F. C., Quantitative studies on the metabolism of tryptophan 
in patients receiving isoniazid therapy. Federation Proc., 1956, 15, 330.

18. deCastro, F. T., Price, J. M., and Brown, R $\mathbf{R}_{0,}$ Reduced triphosphopyridinenucleotide requirement for the enzymatic formation of 3-hydroxylymurenine from L-kynurenine. J. Am. Chem. Soc., 1956, 78, 2904.

19. Walters, C. J., Brown; R. R., Kaihara; M., and Price, J. M., The excretion of $N$-methyl-2-pyridone-5-carboxamide by man following ingestion of several known or potential precursors. J. Biol. Chem, 1955, 217, 489 .

20. Makino, K., Satoh, K. Fujiki, T., and Kawaguchi, K., Relation of 3-hydroxykynurenine to the Ehrlich diazo reaction of urine in severe tuberculosis. $\mathrm{Na}$ ture, 1952, 170, 977.

21. Musajo, L., Spada, A., and Coppini, D, Isolation of 3-hydroxyanthranilic acid from pathological human urine after administration of L-tryptophan. J. Biol. Chem., 1952, 196, 185.

22. Dalgliesh, C. E., and Tekman, S., The excretion of kymurenine and 3-hydroxykynurenine by man. Biochem. J., 1954, 56, 458.

23. Price, I. M., Brown, R. R., Rukavina, J. G., Mendelson, C., and Jahnson, S. A. M., Scleroderma (Acrosclerosis). II Trytophan metabolism before and during treatment by chelation (EDTA). J. Invest. Dermat., In press.

24. Porter, C. C., Clark, I., and Silber, R. H., The effect of $B$ vitamin deficiencies on tryptophan metabolism in the rat. Arch. Biochem., 1948, 18, 339.

25. Henderson, L. M., Weinstock, I. M., and Ramasarma, G. B., Effect of deficiency of B vitamins on the metabolism of tryptophan by the rat. J. Biol. Chem., 1951, 189, 19.

26. Charconnet-Harding, F., Dalgliesh, C. E., and Neuberger, A., The relation between riboflavin and tryptophan metabolism, studied in the rat." Biochem. J., 1953, 53, 513.
27, Mason, M. The metabolism of tryptophan in ribofavin-deficient rats. J. Biol. Chem., 1953, 201, 513.

28. Dalgifesh, C. E.; The relation between thiamine, biotin, and tryptophap metabolism, studied in the rat. Biochem. J., 1955, 61, 328.

29. Know, W. E, Two mechanisms which increase in viad the liver tryptophan peroxidase activity: specific enzyme adaptation and stimulation of the pituitary-adrenal system. Brit. J. Exper. Path., 1951, 32, 462.

30. Dalgliesh, C. E., and Tabechian, H., Comparison of the metabolism of uniformly ${ }^{26} \mathrm{C}$-labelled L-phenylalanine, L-tyrosine, and L-tryptophan in the rat. Biochem. J., 1956, 62, 625.

31. Bray, H. G., Thorpe, W. V., and Wood, P. B, The fate of certain organic acids and amides in the rabbit. 12. Aminohydroxybenzoic acids. Biochem. J., 1951, 48, 394.

32. Henderson, L. M., Quinolinic acid metabolism. $\Pi$. Repfacement of nicotinic acid for the growth of the rat and Neurospora. J. Biol. Chem., 1949, 181, 677.

33. Bokman, A. H, and Schweigert, B. S., 3-Hydroxyanthranilic acid metabolism. III. Molar conversion to quinolinic acid. J. Biol. Chem., 1950, 186, 153.

34. Takahashi, H., Kaihara, M., and Price, J. M., The conversion of kynurenic acid to quinaldic acid by humans and rats. J. Biol. Chem., 1956, 223, 705.

35. Takahashi, $\mathrm{H}_{4}$ and Price, J. M., Urinary excretion of 8-hydroxyquinaldic acid after ingestion of xanthurenic acid. Federation Proc., 1957, 16, 259.

36. Braunshtein, A. E., Goryactienkova, E. V., and Paskina, T. S. Enzymic formation of alanine from L-kynurenine and L-tryptophan, and the role of vitamin $B_{6}$ in this process. Biokhimiya, 1949,. 14, 163.

37. Wiss, O., Der enzymatische Abbau des Kynurenins und 3-Oxy-Kynurenins im tierischen Organismus. Hoppe Seyler's Ztschr. f. physiol. Chem., 1953, 293, 106. 